\title{
Biology and Demography of Creative Potential
}

\author{
Alexander Kholmanskiy \\ Scientific Center BEMCOM, Moscow \\ Laboratory of Medical and Biological Physics \\ allexhol@ya.ru, http://orcid.org/0000-0001-8738-0189
}

\begin{abstract}
The creative potential of homo sapiens is the biological basis of his spirituality. To take into account factor of spirituality in analysis of global problems of demography and ecology used analogies of chemical kinetics. Stable in the historical time, population of people was modeled by open thermodynamic system, equilibrium state of which depends on climate and geophysics. The demography of creative potential was divided into two geographical zones - north and south. Process of society sapientation was formalized by introducing into logistic equation of Verhulst, in addition to a couple of parents, at least one more teacher from among educated people. Stationary solution of the modified kinetic equations determines optimal demography for sustainable development of population in accordance with its education index. The solution of the demographic, energy and environmental problems of mankind is determined by the level of world creative potential, the growth of which is currently limited by the consumption paradigm.
\end{abstract}

Key words: creativity; demography; ecology; north and south; education.

\section{Introduction}

The spiritual man (homo spiritus) is distinguished by the ability to heuristically think and create new information, the worth of which is determined by the measure of increasing and complicating order in the social and material spheres of human existence. Therefore, a fruitful analysis and search for solutions to global problems of energy, ecology and demography are not possible without taking into account the creative potential or spirituality as a key factor of noogenesis [1]. In the present work, the universal laws of chemical thermodynamics were applied to formalize and introduce spirituality into biological and demographic models. The admissibility of these analogies is due to the fact that humanity as a whole is a self-organizing open thermodynamic system, staying in stationary equilibrium in the narrow temperature range $(\mathrm{T}=$ $309-310 \mathrm{~K})$. It is in this range that the anomalous thermodynamic properties of water provide an efficient energy-information exchange of a man with the external environment at the physiological and geocosmic levels $[2,3]$. 


\section{Methods and materials}

Information, as a measure of order, has its energy equivalent, which is formally determined by the product of the system temperature and the negative entropy $(-\mathrm{S})$, called non-entropy [4]. The quantum of external energy initiates in the thermodynamic system, which has cooperative properties, the physico-chemical process of its self-organization, as a result of which the system jumps to a higher level of orderliness of its elements and acquires a new quality. This quality corresponds to a certain portion of new information, the energy equivalent of which increases the free energy of the system. In the external environment, the system must output a portion of thermal energy equal to the product $|\Delta S| T$. The sum of the free energies of all creatively active people determines the current value of the spiritual potential of the society $(\Psi)$ [1]. In the work for the analysis used the current monitoring of the demographic characteristics of the whole world and leading countries [5]. These data are presented in the Table.

Table.

\section{Demography of the world and leading countries as of 04/02/2017}

\begin{tabular}{|c|c|c|c|c|c|}
\hline Specifications & Worldwide & China & India & USA & Russia \\
\hline Total population & $7.51 \times 10^{9}$ & $1.38 \times 10^{9}$ & $1.34 \times 10^{9}$ & $3.26 \times 10^{8}$ & $1.46 \times 10^{8}$ \\
\hline Male population & $3.79 \times 10^{9}$ & $7.09 \times 10^{8}$ & $6.92 \times 10^{8}$ & $1.61 \times 10^{8}$ & $6.78 \times 10^{7}$ \\
\hline Female population & $3.72 \times 10^{9}$ & $6.75 \times 10^{8}$ & $6.48 \times 10^{8}$ & $1.65 \times 10^{8}$ & $7.86 \times 10^{7}$ \\
\hline Born this year & $3.73 \times 10^{7}$ & $4.39 \times 10^{6}$ & $6.82 \times 10^{6}$ & $1.04 \times 10^{6}$ & $4.67 \times 10^{5}$ \\
\hline Died this year & $1.47 \times 10^{7}$ & $2.52 \times 10^{6}$ & $2.48 \times 10^{6}$ & $6.78 \times 10^{5}$ & $5.09 \times 10^{5}$ \\
\hline$\alpha,(\text { man year })^{-1}$ & $1.6 \times 10^{-11}$ & $5.5 \times 10^{-11}$ & $9.1 \times 10^{-11}$ & $2.3 \times 10^{-10}$ & $5.2 \times 10^{-10}$ \\
\hline$\beta,(\text { year })^{-1}$ & $2 \times 10^{-3}$ & $1.8 \times 10^{-3}$ & $2 \times 10^{-3}$ & $2.1 \times 10^{-3}$ & $3 \times 10^{-3}$ \\
\hline Optimal population & $3 \times 10^{9}$ & $7.8 \times 10^{8}$ & $5.3 \times 10^{8}$ & $2.2 \times 10^{8}$ & $1.4 \times 10^{8}$ \\
\hline Education Index, $(\gamma)$ [6] & - & 0.61 & 0.5 & 0.89 & 0.81 \\
\hline Overcrowding ratio & 2.5 & 1.8 & 2.5 & 1.5 & 1.05 \\
\hline
\end{tabular}


In the energy-informational coordinates, the process of sapientation can be adequately described by the dynamics of the accumulation of valuable information or knowledge by the world civilization. The change in the value of $\Psi$ over time characterizes the dependence of the kinetics of sapientation on biosocial and geocosmic factors. It can be assumed that through the action $\Psi$ the Anthropic Principle is realized at the noospheric stage of evolution: Inventiveness is of paramount importance for human evolution. This is the most important product of his creative thinking. The highest goal of human development is the complete domination of consciousness over the material world [7].

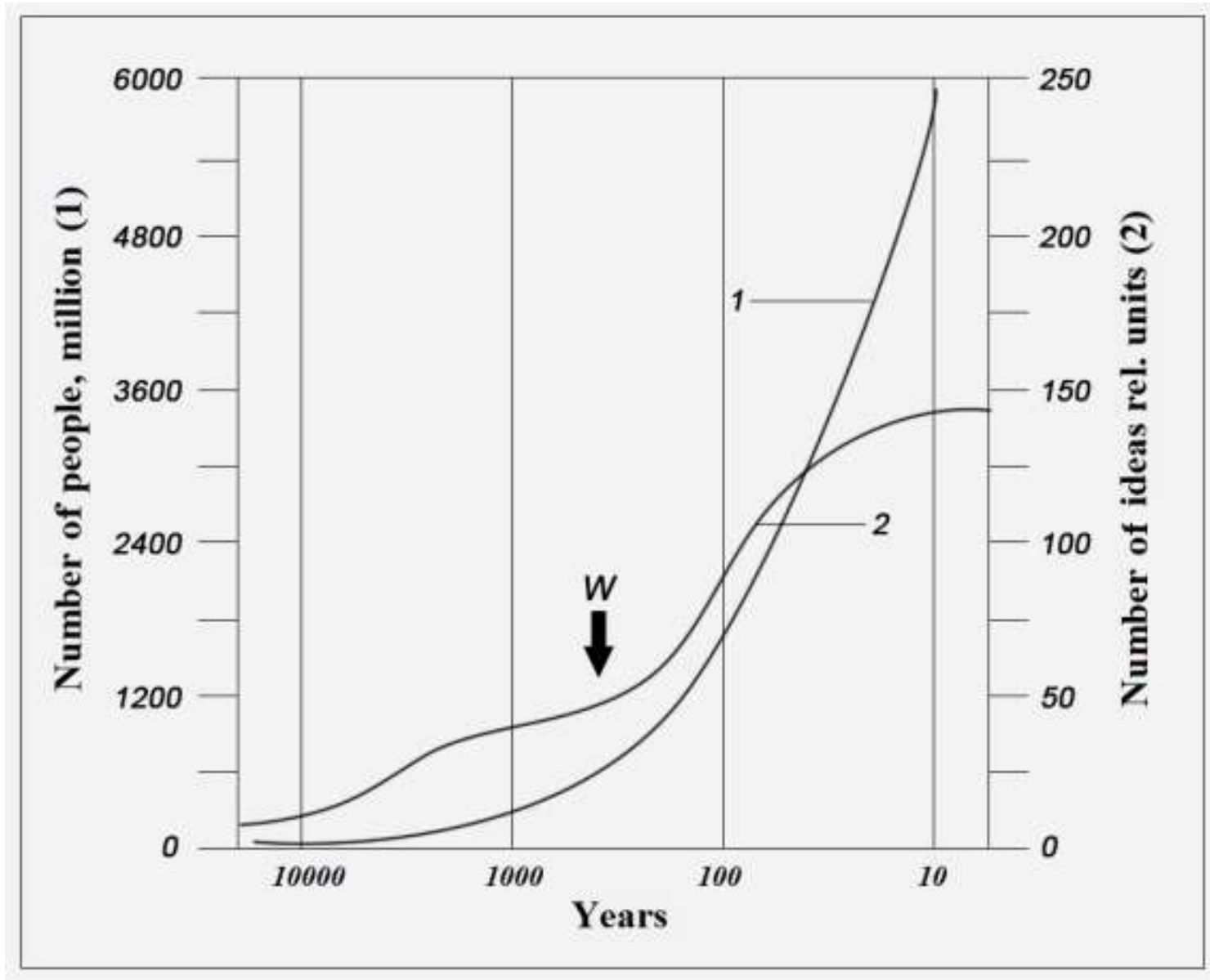

Figure 1. Graphs of the growth of the population of the Earth (1) and the productivity of the creative activity of mankind, summarizing all the valuable ideas, discoveries and inventions at each point in time (2) of [8]. The arrow W marks the beginning of the period when a person uses the canned energy of the sun in the form of hydrocarbons (coal, oil).

The change $\Psi$ in the course of world history at its different stages is illustrated by Figure 1 and Figure 2. From a comparison of the data presented in these figures, it follows that the use of industrial energy of fossil hydrocarbons, first in the form of coal, and then oil and gas, stimulated a sharp acceleration of the spiritualization process. together with the growing number of creators and the entire population. Curve 2 in Figure 1 shows that since the mid-twentieth century, 
population growth has maintained its pace, while the process of sapientation has slowed significantly.

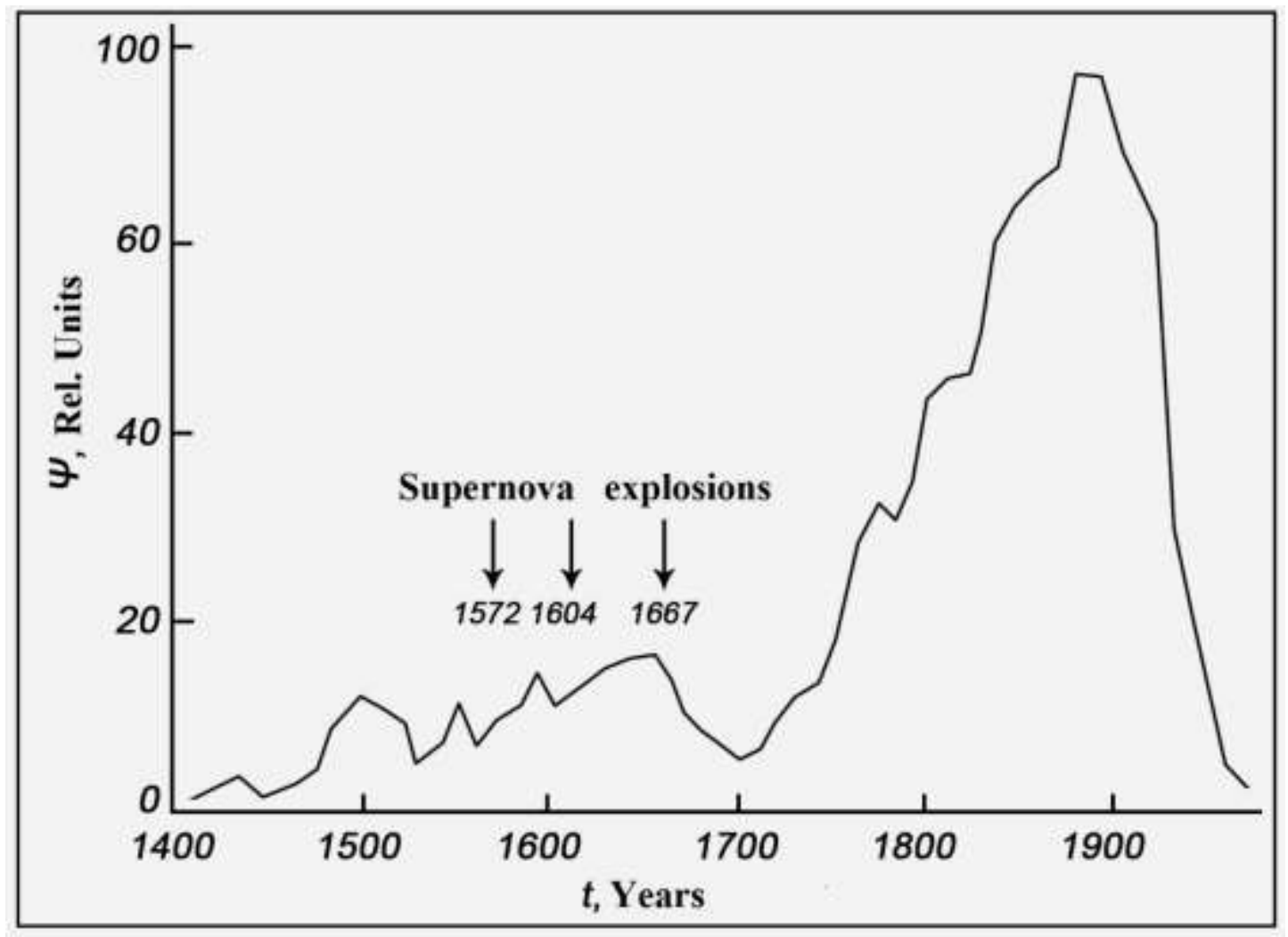

Figure 2. Chronology of changes in the spiritual potential ( $\Psi)$ of the peoples of Europe, proportional to the total creative activity of all the great poets, artists, composers and physicists at every moment of history, from [1]. The arrows indicate the years of supernova explosions.

\section{Demographic model of sapientation}

Representing the system of humanity an open thermodynamic system, the elements and subsystems of which interact with each other at the biosocial level, one can use analogies with chemical kinetics to simulate the demography of spirituality. Note that in existing demographic models, people appear mostly as unreasonable animals that can only reproduce, as long as life resources allow, to fight for territory and resources, and die in their own time. Under such initial conditions, the entire array of global demographic data over many centuries is described with good accuracy by the kinetic equation of the form [9]:

$$
\dot{n}=\alpha n^{2}
$$

where $\mathrm{n}$ is the population size, $\dot{n}$ is its time derivative $(\mathrm{t}), \alpha$ is a constant coefficient with the dimension (year) ${ }^{-1}$.

From (1) follows the hyperbolic dependence of the number of population on t, presented in Figure 1 (curve 1): 


$$
n=\frac{1}{\alpha\left(t-t_{\Omega}\right)} \text {. }
$$

In (2), $t_{\Omega}$ is the conditional collapse time, when $\mathrm{n} \rightarrow \infty$, and vital resources, territorial and food, tend to zero. A demographic model that takes into account the finiteness of a life resource is described by the logistic growth equation or the Verhulst equation:

$$
\frac{d n}{d t}=n\left(r-\frac{r}{K} n\right)
$$

where $r$ is a constant with the dimension of the specific growth rate of the population, $K$ is the limiting capacity of an ecological niche characterizing food and territorial resources. When $\mathrm{K} \gg \mathrm{r}$, from (3) follows an exponential dependence of the population on t. The negative sign of the second term in (3) is interpreted as the contribution of mortality to the population dynamics.

Note that the kinetic equations similar to (1) and (3) can be compiled to describe the dynamics of a population of unicellular cannibals or a mixed predator-prey population [9]. These biomodels are suitable for describing the demographics of human populations living under archaic religious or dictatorial regimes.

In [9], equation (1) was given a biological interpretation, suggesting that the growth rate of the population growth rate of the childbirth reaction, in which the male and female individuals participate, is limited. The energy of the reaction is provided by vital energy, the action of which is manifested in the form of sexual instinct. The number of men $\left(\mathrm{n}_{+}\right)$and women $\left(\mathrm{n}_{-}\right)$are approximately equal to $\mathrm{n} / 2$ (see Table), while the shares of men and women of reproductive age are $\sim 1 / 2$ from $n_{+}$and and $\sim 1 / 3$ from $n_{-}[5,10]$.

Taking into account these data and the rate of decrease in abundance due to mortality $(\beta n)$, equation (1) is transformed into a second-order kinetic equation, conventionally called antilogistic [9]:

$$
\frac{d n}{d t}=\frac{\alpha}{6}\left(n_{+} n_{-}\right)-\beta \mathrm{n}=\frac{\alpha}{24}\left(n^{2}\right)-\beta \mathrm{n} .
$$

In (4), $\alpha$ and $\beta$ are effective constants of the rate of birth and death. The current values of $\alpha$ and $\beta$ are estimated using (4) and the data in the Table. At the same time, the number of people born and died in a year was equated with the expressions $\frac{\alpha}{6}\left(n_{+} n_{-}\right)$and $\beta \mathrm{n}$, respectively. The obtained values of $\alpha$ and $\beta$ are listed in the Table. Note that the $\alpha$ estimate for the whole world agrees well with the magnitude of the probability of having a child within a year of a selected pair, which for many centuries was $2 \times 10^{-11}$ [9].

Optimal for sustainable development, the population size $\left(\mathrm{n}_{\mathrm{o}}\right)$ in the world and in different countries will be determined by the zero rate of population growth at $\alpha$ and $\beta$ values corresponding to a given time: 


$$
n_{o}=\frac{24 \beta}{\alpha}
$$

The obtained values of $n_{o}$ are given in the Table. Dividing by $n_{o}$ the current population, it is possible to determine the overpopulation coefficients of the planet and countries currently relevant (Table). Comparison of the obtained values shows that the main responsibility for the overpopulation of the planet lies with countries located in the climatic zone below $\sim 40^{\circ} \mathrm{nl}$ and above $40^{\circ} \mathrm{sl}$ (India, China and other countries in South America, Africa and Asia).

The rate constant for the creator's $\left(\alpha_{*}\right)$ birth reaction in Europe during the 15th-19th centuries. can be estimated similarly to the value of $\alpha$ using the graph of the change in the number of creators in Figure 2 and the order of the population size $\sim 10^{8}[1]$. With the number of creators of the order of 10-100, the value of $\alpha_{*}$ will be $10^{-14}-10^{-13}$ (man year) ${ }^{-1}$, which is $3-4$ orders of magnitude lower than the current value of $\alpha$.

The activity of creators at the early stage of spiritualization was associated with the generation of new knowledge and an increase in the proportion $(\gamma)$ of the educated population (Figure 3), the number of which we denote by $n_{*}$. The growth rate $n_{*}$ can be described by equation (4), complementing the birth reaction with the subsequent training of a person. The level of education of the person will correlate with the number of teachers $(m)$ involved in the learning process. Representing $n_{*}=\gamma \mathrm{n}$ and taking the constant mortality rate constant for $n_{*}$, we obtain an analogue of equation (4):

$$
\frac{d n_{*}}{d t}=\frac{\alpha}{6}\left(n_{+} n_{-}\right) n_{*}^{m}-\beta n_{*}=\frac{\alpha}{24 \gamma^{2}}\left(n_{*}^{m+2}\right)-\beta n_{*}
$$

From $\left(4^{*}\right)$ for the stationary state of the population that corresponds to sustainable development follows the optimal number of educated people:

$$
n_{*}^{o}=\sqrt[m+1]{\gamma^{2} n_{o}}, \mathrm{~m}=1,2,3,4 .
$$

The number $m$ determines the level of education: primary $(m=1)$, medium $(m=2)$, highest $(m=3)$, scientific $(m=4)$. The total number $n_{*}$ characterizes the index of education of countries given in the Table.

To date, the process of spiritualization has reached a plateau (Figure 1) and the number of great creators has dropped to almost zero (Figure 2). Such a state of world civilization is a consequence of the globalization of the consumption paradigm [11]. Accordingly, the world energy, ecology and demography turned out to be hostages of the teleology of the modern world view, the ideology of which has not yet been cleared of religious rudiments and the mystics of fundamental physics [1, 12].

\section{Energy process of sapientation}


The representation of mankind as a thermodynamic open system allows using the analogies of chemical kinetics to introduce the factor of spirituality into demographic models. It is mainly about energy models of the process of spiritualization in the period starting from point $t_{w}$ in Figure 1 to the conditional point $t_{\Omega}$, in the vicinity of which the process of sapientation begins to stagnate due to the globalization of the consumerism paradigm (Figure 1).

Obviously, the growth of the potential of the noosphere $\Psi$ in the process of spiritualization on the interval $t_{W}-t_{\Omega}$ will correlate with the rate of decrease of fossil hydrocarbons. We associate their potential energy with the solar energy conserved in the bowels of the earth during geological epochs and denote $U_{\odot}$, and the current energy of solar radiation incident on the Earth is $E_{\odot}$.

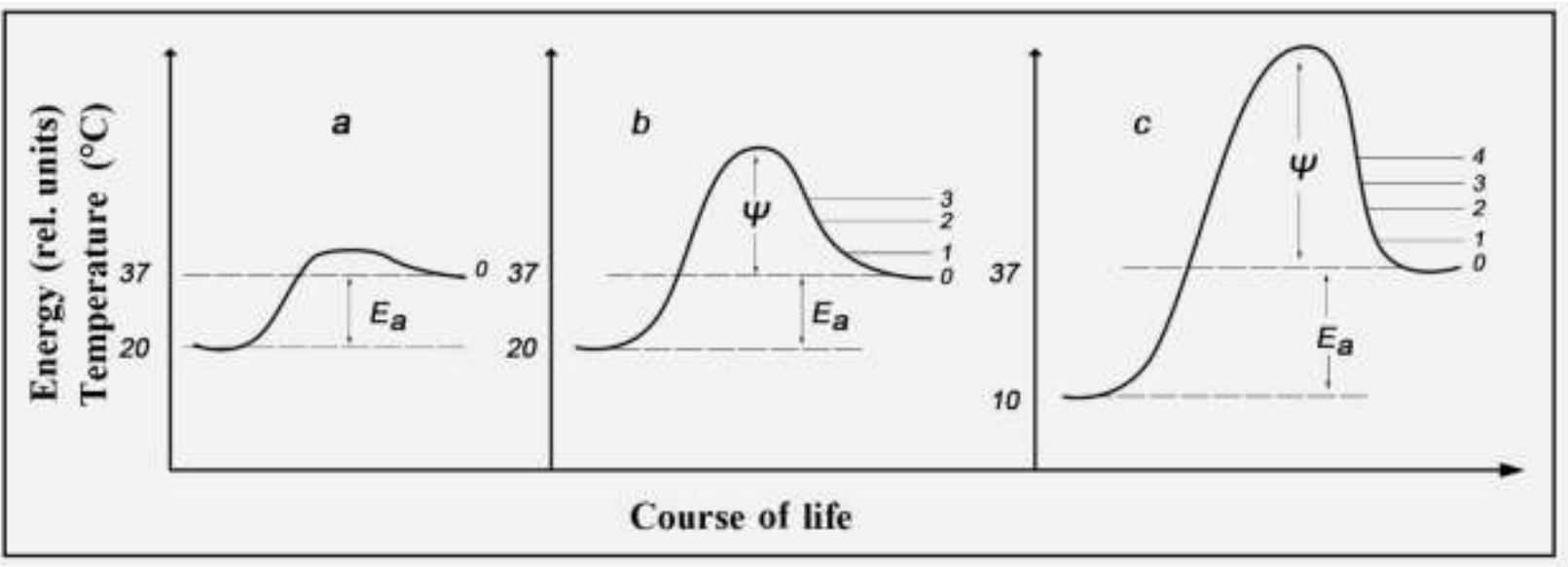

Figure 3. Energy of life of primates (a), population of latitudes of the B-zone $\sim 40^{\circ} \mathrm{nl} \sim 40^{\circ} \mathrm{sl}(\mathrm{b})$ and $C$-zones $\sim 60^{\circ} \mathrm{nl} \sim 40^{\circ} \mathrm{nl}(\mathrm{c}) . E_{a}$ is the vital energy of activation of life processes, $\Psi$ is the spiritual potential of the noosphere, numbers denote different levels of education.

Figure 3 shows the layout of the energy states of the external environment and man for two temperature zones where people live, in selected latitudes $\sim 40^{\circ} \mathrm{nl} \sim 40^{\circ} \mathrm{sl}$ (B-zone) and $\sim 60^{\circ} \mathrm{nl}$ $\sim 40^{\circ} \mathrm{nl}$ (C-zone). For comparison, a diagram is given for a primate - a great ape. Vital energy $\left(E_{a}\right)$, electromagnetic in nature, enters the body with food and water and its magnitude is proportional to the current energy of the sun $E_{\odot}$ and energy $U_{\odot}$, converted by man into food and fuel:

$$
E_{a}=\varphi_{1} \mathrm{E}_{\odot}+\varphi_{2} U_{\odot}
$$

where $\varphi_{1}$ and $\varphi_{2}$ are the efficiency factors of energy utilization methods. The energy $\varphi_{1} \mathrm{E}_{\odot}$ at the stage of evolution to $t_{W}$ was quite enough for the life and reproduction of populations living mainly in the B-zone. During human settlement throughout the Earth, the difference in the climate of the B- and C-zones led to the following relationships:

$$
\mathrm{E}_{\overparen{C}}^{\sigma}>\mathrm{E}_{\overparen{C}}^{\mathrm{B}} ; \mathrm{E}_{\mathrm{a}}^{\sigma}<\mathrm{E}_{\mathrm{a}}^{\mathrm{B}} ; \varphi_{1}^{\sigma}>\varphi_{1}^{\mathrm{B}} ; \varphi_{2}^{\sigma}<\varphi_{2}^{\mathrm{B}} .
$$


The fulfillment of the relation $E_{a}^{\sigma}<E_{a}^{B}$ in (6) stimulated the development of science necessary for developing the ways of energy $U_{\odot}$ in $E_{a}$, mainly in the B-zone, therefore $\varphi_{2}^{\sigma}<\varphi_{2}^{\mathrm{B}}$.

Using the analogy with the Arrhenius equation [6], the multiplication rate constant from equation (4) is represented by the exponent:

$$
\operatorname{Aexp}\left(-\frac{\mathrm{E}_{\mathrm{a}}}{k T}\right)
$$

where $\mathrm{A}$ is a constant characterizing the mating frequency, $\mathrm{k}$ is the Boltzmann constant, and $\mathrm{T}$ is the absolute temperature. Then, with an equal number of populations, increased congestion and $\mathrm{E}_{\mathrm{a}}^{b}<\mathrm{E}_{\mathrm{a}}^{c}$ ratio in the $\mathrm{B}$-zone will lead to a higher population growth rate than in the C-zone.

If we denote $U_{\odot}^{a}$ share of $U_{\odot}$, addressed to $E_{a}$, then the energy problem of spiritualization at the current moment can be represented by a striving for the ratio unit $U_{\odot}^{a} / U_{\odot}$ and a real threat of exhaustion of $U_{\odot}$. The solution to this problem should combine:

1) reducing $U_{\odot}^{a}$ by optimizing the size of the population first of all in the B-zone and demilitarizing the world economy;

3 ) the growth of the potential of the noosphere $\Psi$ due to the approach of the parameter $\gamma$ to 1 in the B-zone and the parameter $m$ to 3, 4 in the C-zone;

4) approximation of the values of $\varphi_{1}$ and $\varphi_{2}$ to 1 for irreversible and renewable energy, as well as the development of alternative energy methods for producing energy [13, 14].

The method of analogies with chemical kinetics and thermodynamics, proposed to take into account the factor of spirituality, allows to construct a model of the sustainable development of mankind and satisfactorily describing the demography of its creative potential.

\section{References}

1. Kholmanskiy, A.S. Real Spirituality. World of Science. 2014. (2). http://mirnauki.com/PDF/11FILSMN214.pdf

2. Kholmanskiy, A.S. Chirality and quantum effects as factors of morphogenesis. Electron. Math. Med.-Biol. J. 2010. 9(4) URL: http://www.sci.rostelecom67.ru/user/sgma/MMORPH/N-28html/kholmanskiy-2/kholmanskiy-2.htm

3. Kholmanskiy, A., Zaytseva, N. Physically adequate approximations for abnormal temperature dependences of water characteristics. J. Mol. Liq. 2019 (275) 741-8. https://doi.org/10.1016/j.molliq.2018.11.059

4. Blumenfeld, L.A. Solvable and unsolvable problems of biological physics. URSS. 2002. $160 \mathrm{p}$.

5. Counter population of the Earth, 04/02/2017. http://countrymeters.info/ru/World

6. Rating of countries of the world by level of education

http://gtmarket.ru/ratings/education-index/education-index-info . 
7. Tesla, N. Articles. Samara. 2008. http://library.raikevich.com/tesla/index 1.html\#00

8. Bogdankevich, O. V., Lectures on ecology. M. 2002. 206 p.

9. Nurgaliev I.S. The inevitability of nonlinearity: the antilogistic "nature of the demographic process. International Scientific and Practical Conference "Information Technologies in Education and Science" (ITON-2016). Collection of papers. Kazan (Volga Region) Federal University. November 5 - 7, 2016. 152-161

10. Apolikhin, O.I., Moskaleva, N.G., Komarova, V.A. The current demographic situation and the problems of improving the reproductive health of the population of Russia; http://ecuro.ru/node/3567

11. Baudrillard, J. Consumer Society. His myths and structures. M . Republic, 2006.

12. Nizovtsev, V.V. Time and place of physics of the XX century. URSS. 2000. 201 p.

13. Bezrukikh, P.P., Strebkov, D.S., Kholmanskiy, A.S. Perspective directions for the development of renewable energy sources. Vestnik VIESH. 2016. 4. 96-101.

14. Kholmanskiy A.S., Strebkov D.S. Energy of the noosphere. Russian Agricultural Sciences. 2004 (1) 44-47. 